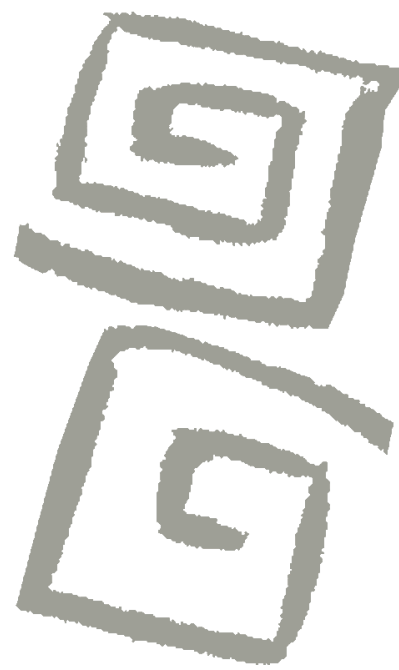

\title{
Hombres heterosexualmente activos, masculinidades, prevención de infección por VIH y búsqueda de profilaxis posexposición sexual consentida
}

\author{
Heterosexually active men, masculinities, HIV \\ prevention, and the search for post-exposure \\ prophylaxis following sexual exposure
} Lorruan Alves dos Santos ${ }^{1}$, Marcia Thereza Couto ${ }^{2}$, Augusto Mathias ${ }^{3}$,
Alexandre Grangeiro ${ }^{4}$

${ }^{1}$ Fisioterapeuta, Magíster en Salud Colectiva. Estudiante de doctorado en Salud Colectiva, Faculdade de Medicina, Universidade de São Paulo, São Paulo, Brasil. $\bowtie$ (iD)

${ }^{2}$ Doctora en Sociología Posdoctora en Salud Colectiva. Profesora, Departamento de Medicina Preventiva, Faculdade de Medicina, Universidade de São Paulo, São Paulo, Brasil. $\triangle$ (iD)

${ }^{3}$ Magíster en Ciencias (Medicina Preventiva). Estudiante de doctorado en Salud Colectiva, Faculdade de Medicina, Universidade de São Paulo, São Paulo, Brasil. $\square$ (iD)

${ }^{4}$ Cientista Social. Investigador, Departamento de Medicina Preventiva, Faculdade de Medicina, Universidade de São Paulo, São Paulo, Brasil. \ iD
RESUMEN La masculinidad hegemónica distancia a los hombres heterosexuales activos de las respuestas a la epidemia de sida. Sin embargo, con el paradigma de la prevención combinada, la profilaxis posexposición sexual hace posible nuevas formas de gestionar los riesgos de infección, al tiempo que trae nuevos desafíos. El objetivo de este artículo es, a la luz de la perspectiva de género y de las masculinidades, discutir cómo los hombres heterosexuales activos perciben su riesgo frente al $\mathrm{VIH}$, cómo manejan los métodos y estrategias de prevención, incluyendo la profilaxis posexposición sexual. El estudio de abordaje cualitativo entrevistó 16 hombres heterosexuales activos en cinco servicios de salud de diferentes ciudades brasileñas. El análisis señala que el uso del condón, principal método de prevención, fue condicionado a factores subjetivos y contextuales; y su fallo se destaca como el principal motivo de búsqueda de la profilaxis posexposición sexual. Los ideales de masculinidad hegemónica penetraron los discursos de percepción y gestión de riesgo en el actual contexto de la epidemia de sida.

PALABRAS CLAVES Masculinidad; Heterosexualidad; Salud del Hombre; Prevención de Enfermedades; VIH; Profilaxis Posexposición; Brasil.

\begin{abstract}
Hegemonic masculinity has distanced heterosexually active men from the responses to the HIV epidemic. However, with the combined prevention paradigm, postexposure prophylaxis offers new ways to manage the risks of HIV infection, while at the same time bringing about new challenges. The aim of this paper is to discuss - through the lens of gender and masculinities - how heterosexually active men perceive their HIV risk and how they manage prevention methods and strategies, including post-exposure prophylaxis. Employing qualitative methods, 16 heterosexually active men in five health services from different Brazilian cities were interviewed. The analysis indicates that condom use - the main prevention method - was conditioned by subjective and contextual factors, and its failure stands out as the primary reason for seeking out post-exposure prophylaxis. We argue that ideals of hegemonic masculinity permeate perceptions and risk management discourses in the current context of the HIV/AIDS epidemic.
\end{abstract}

KEY WORDS Masculinity; Heterosexuality; Men's Health; Disease Prevention; HIV; PostExposure Prophylaxis; Brazil. 


\section{INTRODUCCIÓN}

Actualmente, la epidemia de VIH-sida se comporta de manera bastante heterogénea entre diferentes regiones del planeta y hasta dentro de un mismo país, según los contextos locales y sus grupos poblacionales. En Brasil, por ejemplo, en los casos de VIH notificados en el Sistema Nacional de Vigilancia Epidemiológica, los hombres son mayoría. Si bien, desde el inicio de la epidemia, se ha producido una diminución de la razón entre ambos sexos, esta es aún bastante alta para los hombres. En 2014, la razón era de una mujer por cada 2,2 hombres y, en 2017, Ilegó a una mujer por cada 2,6 hombres. Entre 2014 y 2017, los datos registrados mostraban $150.493 \mathrm{ca}-$ sos notificados, de los cuales el $70,6 \%$ eran hombres y el $29,4 \%$ mujeres $^{(1)}$.

En lo referente al tipo de exposición, los datos mundiales estiman que el $70 \%$ de los casos de infección por VIH son por transmisión heterosexual ${ }^{(2)}$. En Brasil, estetipo de transmisión también se presenta como el principal medio de infección de $\mathrm{VIH}$ en las mujeres y como la segunda mayor categoría de exposición para los hombres, con el 36,9\% de los casos. Las transmisiones homosexual, bisexual y por uso de drogas inyectables fueron del $49,9 \%$, el $9,5 \%$ y el $2,6 \%$, respectivamente ${ }^{(1)}$.

Si bien es bastante razonable suponer que tanto hombres como mujeres heterosexualmente activos necesitan ser considerados de modo ecuánime en las intervenciones para prevenir la infección por $\mathrm{VIH}$, en la práctica sucede poco, incluso cuando los datos epidemiológicos son claros en lo referente al lugar ocupado en el contexto de la epidemia ${ }^{(3,4)}$.

Los hombres heterosexualmente activos -considerados como aquellos que, independientemente de mantener o no prácticas sexuales con otros hombres, se autodeclararon heterosexuales y afirmaron que mantenían prácticas sexuales con mujeres ${ }^{(2)}$ - aún siguen siendo tratados por ciertos informes y documentos, por ejemplo de organismos gubernamentales de instituciones internacionales, de manera genérica y dando poca relevancia a los contextos que pueden colocarlos en situaciones de vulnerabilidades. Por otro lado, en los casos en los que sí se observa mayor atención, se exploran poco las diferenciaciones internas del segmento, según marcadores sociales como raza, clase y generación ${ }^{(2,4,5)}$.

Con relación a la tendencia de invisibilidad de los hombres heterosexualmente activos en las políticas e investigaciones en el campo del $\mathrm{VIH}$-sida, recientes estudios empíricos y debates conceptuales en el campo de las masculinidades, fundamentados en la perspectiva constructivista, relacional y feminista de género, han contribuido fuertemente a la revisión de esta tendencia al postular que las normas de género desempeñan un papel importante en el moldeado del comportamiento sexual y contribuyen a la comprensión de las prácticas masculinas en diferentes contextos de experiencias de la sexualidad, la socialización y la formación de identi$\operatorname{dad}^{(2,3,6,7)}$. Esta agenda reciente de investigación en el campo de la salud pública, en articulación con las ciencias sociales, ha ayudado a evitar el uso de una noción genérica de "hombre" como categoría predominante en los estudios biomédicos, como también ha buscado investigar empíricamente las diferentes maneras por las cuales los hombres ejercitan la masculinidad y cómo eso se relaciona con los procesos de salud, enfermedad y cuidado.

Comprender la evolución de la epidemia de VIH-sida desde la década de 1980 hasta la actualidad, como también las representaciones colectivas involucradas, es bastante ilustrativo acerca de cómo los ejes estructurantes de la masculinidad hegemónica influyen en la forma en que los hombres heterosexuales reconocen y lidian con cuestiones relacionadas a la salud y, particularmente, con las evaluaciones del riesgo y la prevención de enfermedades sexualmente transmisibles y el VIH.

En el ámbito de los ideales colectivos, el orden de género que origina y diferencia las masculinidades de las feminidades está atravesado por una jerarquía fuertemente anclada en preceptos patriarcales. En esa misma lógica, la masculinidad hegemónica no es solo hegemónica en relación con las otras mascu- 
linidades subalternas y marginalizadas, sino a todo orden de género. En otras palabras, la masculinidad hegemónica expresa relaciones de poder ${ }^{(8,9)}$.

Gomes $^{(9)}$ sintetiza las premisas que validan la masculinidad hegemónica: a) parten de los ideales del patriarcado, que reconoce a los hombres como superiores a las mujeres; b) no está relacionada directamente con el poder económico de ciertas personas, sino un modelo -o sea, un ideal- de masculinidad; c) guía la manera en que los hombres se relacionaron con las mujeres y naturaliza las jerarquías de género; d) no es un modelo fijo y constante, lo que permite adecuaciones culturales y situacionales; y) es una posición que debe ser conquistada y no dada o pasada de una persona a otra, como otros sistemas de opresión y; f) precisa ser analizada una estructura más amplia y considerando otras variables y marcadores sociales. Además de eso, la fuerza física y mental, la autonomía, la disposición para trabajar y la propia heterosexualidad son entendidas como ejes estructurantes y que sustentan el concepto de masculinidad hegemónica.

Los patrones hegemónicos de masculinidad, en asociación a la heteronormatividad, poseen una fuerte influencia en la evaluación personal del riesgo, ya que esta se conforma en la posición que los sujetos ocupan en la estructura social, los elementos contextuales de los ambientes en los cuales transitan, los significados que atribuyen a las experiencias pasadas, las relaciones que establecen entre pares y con otros grupos de pertenencia y las posibilidades de acceso y el uso que hacen de la información, los insumos y los servicios. En términos ilustrativos para la evaluación personal de riesgo de infección por VIH, la misma acción (penetración anal, por ejemplo) puede despertar percepciones diferentes de peligro dependiendo de los individuos involucrados (matrimonio homosexual masculino o heterosexual, por ejemplo), del escenario (calle, hotel o en casa, por ejemplo) y de otros elementos presentes al momento de la exposición (uso de alcohol y drogas, por ejemplo). Siguiendo la misma lógica, si la pareja en el momento de la exposición fuera eventual o profesional del sexo, el riesgo o peligro será percibido de manera distinta al riesgo percibido cuando la exposición es con una pareja fija.

Ante la complejidad que involucra las percepciones y evaluaciones personales de riesgo de infección por $\mathrm{VIH}$, actualmente ningún método o estrategia de prevención aplicado de forma aislada es eficaz o suficiente para el control de la epidemia ${ }^{(10,11)}$. Así, la prevención combinada se configura como una apuesta importante de organismos nacionales e internacionales. Según el Programa Conjunto de las Naciones Unidas sobre el VIH-sida (ONUSIDA), ese nuevo modelo orientador de prácticas y acciones de prevención de la infección por VIH pretende tener un impacto más duradero en la reducción de la incidencia de nuevos casos de infección. En síntesis, esa estrategia consistiría en el uso combinado de intervenciones basadas en evidencias que articulan tecnologías biomédicas, agendas comportamentales e intervenciones estructurales más amplias, en el ámbito individual y colectivo, con acciones sensibles a las demandas y especificidades de los sujetos y sus contextos, sin perder de vista la dinámica de la transmisión del $\mathrm{VIH}^{(11,12,13)}$.

Entre las opciones disponibles en el abanico de la prevención combinada, se destacan los métodos biomédicos basados en terapia antirretroviral, como la profilaxis preexposición sexual y la profilaxis posexposición sexual consentida. Estos métodos consisten en el uso de antirretrovirales por vía oral, tópica o intravenosa -antes o después de la exposición al virus- a fin de evitar la seroconversión por personas seronegativas ${ }^{(14)}$. En Brasil, desde 2010, la profilaxis posexposición sexual pasó a ser prescrita para situaciones en las que la exposición se dio por vía sexual consentida ${ }^{(15,16)}$.

Diversos estudios y protocolos clínicos nacionales e internacionales recomiendan el inicio de la profilaxis con antirretrovirales lo más precozmente posible, con un plazo de hasta 72 horas luego de la exposición ${ }^{(15,17,18)}$. Los tipos de exposición con riesgo de transmisión del VIH indicados al uso de la profilaxis posexposición sexual son aquellos que 
presentan contacto con la sangre, el semen, los fluidos vaginales, los líquidos de serosas, el líquido amniótico, el líquido cefalorraquídeo, el líquido sinovial y la leche materna. El esquema profiláctico preferencial es el que combina el tenofovir con lamivudina y dolutegravir. Según el protocolo clínico del Ministerio de Salud, ese esquema posee menor número de efectos adversos y baja interacción medicamentosa, lo que propicia mejor la adhesión y el manejo clínico ${ }^{(15)}$.

$\mathrm{Si}$ conderamos que estudios recientes han mostrado que el acceso a los conocimientos sobre los métodos basados en antirretrovirales -sobre todo la profilaxis posexposición y preexposición sexual- es desigual entre categorías de género y orientación sexual; que los hombres que tienen sexo con hombres poseen hasta dos veces más información sobre tales métodos de prevención en comparación con los hombres heterosexuales y las mujeres en general ${ }^{(19,20)}$; que la evaluación del riesgo personal relacionado con el VIH está conformada por las posiciones de los sujetos según referenciales de género, orientación sexual; y que en los estudios en el campo de la prevención al VIH-sida se investiga poco a los hombres con prácticas heterosexuales, en este artículo buscamos analizar las dimensiones de la prevención y del riesgo y la búsqueda de profilaxis posexposición sexual entre hombres heterosexualmente activos. En otras palabras, el objetivo de este artículo es discutir cómo los hombres heterosexualmente activos perciben su riesgo personal frente al $\mathrm{VIH}$, cómo gerencian los métodos y estrategias utilizados antes de buscar profilaxis posexposición sexual, así como comprender el contexto de la exposición que motivó la búsqueda y el uso de la profilaxis, a la luz de la perspectiva teórica de género y masculinidades.

\section{METODOLOGÍA}

Los datos empíricos de este trabajo forman parte de una investigación mayor ${ }^{(21)}$ de abordaje cuantitativo y cualitativo, titulada " $A$ efetividade da profilaxia da transmissão do
HIV pós exposição sexual consensual, do uso combinado dos métodos preventivos contra a infecção pelo HIV e a profilaxia pré exposição sexual, em serviços públicos brasileiros", que investiga la efectividad de la profilaxis de la transmisión del VIH posexposición sexual consensuada en servicios de salud públicos de cinco ciudades pertenecientes a tres regiones de Brasil: en la región sur, la ciudad de Porto Alegre (estado de Rio Grande do Sul) y la ciudad de Curitiba (estado de Paraná); en la región nordeste, la ciudad de Fortaleza (estado de Ceará); y en la región sudeste, las ciudades de Ribeirão Preto y São Paulo (estado de São Paulo).

El estudio fue llevado a cabo bajo los lineamientos establecidos en la Resolución $\mathrm{N}^{\circ}$ 466, de diciembre de 2012, por la Comisión Nacional de Ética en Investigación del Consejo Nacional de Salud, y evaluada y aprobada por la Comisión de Ética en Investigación de la Facultad de Medicina de la Universidad de San Pablo, bajo el parecer 251/14.

En diálogo con la investigación mayor, el recorte propuesto en este trabajo pone énfasis en el segmento de los hombres heterosexualmente activos en el marco de una investigación cualitativa. Se partió de entender que este abordaje "busca alcanzar el universo de los significados, de los motivos, de las aspiraciones, de las creencias, de los valores y de las actitudes"(22). Para la producción de los datos empíricos se empleó la técnica de entrevistas narrativas. Al trabajar con este tipo de modalidad (narrativa), se observa que los relatos de las experiencias narradas son representados y recontados, los eventos siguen un orden significativo coherente que permite la articulación entre pasado, presente y futuro. Se utiliza información que ocurre en el pasado con la mirada del presente y la proyección del futuro ${ }^{(23)}$.

La participación en el estudio siguió los siguientes criterios de inclusión: ser hombre cisgénero y heterosexualmente activo, con edad igual o superior a 18 años; haber consultado el servicio participante para la realización del test de $\mathrm{VIH}$ y/o la profilaxis como consecuencia de una exposición sexual; que el test de VIH haya resultado negativo al momento de consultar el servicio; haber usado 
profilaxis posexposición sexual en el servicio, excluyendo el uso de la profilaxis para fines de reproducción asistida, exposición ocupacional o violencia sexual. Los participantes del estudio fueron convocados en distintos momentos del contexto de atención en los servicios participantes de la investigación: en la prescripción de la profilaxis, durante el uso de la profilaxis (en los días entre la prescripción hasta el día 28) y en los retornos periódicos propuestos como forma de seguimiento pos uso de la medicación (período hasta seis meses posteriores a la fecha de la prescripción de la profilaxis).

A partir de las vivencias afectivo-sexuales de los entrevistados, a través de la guía de entrevistas se pretendió investigar la relación entre las prácticas sexuales y la búsqueda y adopción de la profilaxis posexposición sexual, teniendo como foco la manera por la cual estos hombres gerencian el propio riesgo de exposición al VIH.

Todas las entrevistas se realizaron en ambientes privados, al interior de los servicios participantes, en el período de marzo a diciembre de 2015 y se llevaron a cabo por investigadores previamente entrenados y familiarizados con la guía y bajo la supervisión de la coordinación de la investigación, luego de la lectura, concordancia y firma de los términos del consentimiento libre e informado, por parte de los participantes. El tiempo de duración promedio de cada entrevista fue de 45 minutos y se audiograbaron digitalmente. Se transcribieron los audios en su totalidad y se utilizó el software QSR Nvivo ${ }^{\circledR}$ para auxiliar en la organización, agrupamiento temático de los relatos, como también el tratamiento de los datos, con base en las categorías empíricas previamente establecidas.

Para el análisis de los datos empíricos utilizamos el método de interpretación de sentidos, basado en principios hermenéuticos-dialécticos, que apunta a interpretar el contexto, las razones y las lógicas de falas, acciones e interrelaciones entre grupos e instituciones ${ }^{(24)}$. En la trayectoria analítico-interpretativa se recorrieron los siguientes pasos: a) lectura comprensiva, visando impregnación, visión de conjunto y aprehensión de las particularidades del material de la investigación; b) identificación y recorte temático que emergieron de los relatos; c) identificación y problematización de las ideas explícitas e implícitas; d) busca de sentidos más amplios (socioculturales), subyacentes a las falas de los sujetos de la pesquisa; e) diálogo entre las ideas problematizadas, informaciones provenientes de otros estudios acerca del asunto y los referenciales conceptuales del estudio, género y masculinidades; y f) elaboración de síntesis interpretativa, procurando articular objetivo del estudio, bases teóricas adoptadas y datos empíricos producidos.

Dada la diversidad de las maneras de pensar y prevenir la infección por VIH, incluyendo el uso de la profilaxis posexposición sexual, propusimos organizar y analizar las categorías temáticas apuntando a destacar las similitudes, diferencias y contradicciones en los relatos de los participantes. Entendemos, también, que los modos de gerenciar los riesgos y las motivaciones en la búsqueda de profilaxis posexposición sexual son particulares a las significaciones personales $y$, al mismo tiempo, están ligados a contextos personales y socialmente dados. Por todo eso, buscamos destacar en el análisis las imbricaciones y los cruces de lo social, expresados por los diferentes marcadores sociales que posicionan y tensionan los sujetos en el mundo.

\section{RESULTADOS Y DISCUSIÓN}

La edad de los entrevistados varió entre 18 y 58 años, con una media de 33,8 años. La mayoría de los participantes se autodeclaró como blancos $(n=9)$, cristianos $(n=11)$, sin hijos $(n=10)$ y tenía pareja casual en el momento de la entrevista $(n=8)$. En lo que se refiere al tipo de pareja en el momento de la exposición, 14 fueron con parejas casuales de serología desconocida para $\mathrm{VIH}$, y dos con pareja estable, uno con pareja estable con serología desconocida y otro con pareja serodiscordante (Tabla 1).

Con relación a los ejes de análisis, en primer término, se presentan y discuten los 
Tabla 1. Características sociodemográficas de los hombres heterosexuales activos entrevistados.

\begin{tabular}{|c|c|c|c|c|c|c|c|c|c|}
\hline Nombre & $\begin{array}{l}\text { Edad } \\
\text { (años) }\end{array}$ & Color & Lugar & $\begin{array}{l}\text { Tipo de } \\
\text { exposición }\end{array}$ & Religión & $\begin{array}{l}\text { Relación } \\
\text { estable }\end{array}$ & Hijos & $\begin{array}{l}\text { Categoría de } \\
\text { profesión }\end{array}$ & $\begin{array}{l}\text { Trabajo } \\
\text { formación }\end{array}$ \\
\hline Pablo & 18 & Blanca & RIB & $\begin{array}{l}\text { Pareja } \\
\text { casual }\end{array}$ & $\begin{array}{c}\text { Cristiano o cree en } \\
\text { Dios }\end{array}$ & No & No & $\begin{array}{l}\text { Profesionales } \\
\text { de las ciencias } \\
\text { y de las artes }\end{array}$ & $\begin{array}{l}\text { Profesor/ } \\
\text { voluntario }\end{array}$ \\
\hline Iván & 22 & Parda & SP & $\begin{array}{l}\text { Pareja } \\
\text { casual }\end{array}$ & Católico no praticante & No & No & Comercio & Mozo \\
\hline Dario & 23 & Branca & CUR & $\begin{array}{l}\text { Pareja } \\
\text { casual }\end{array}$ & $\begin{array}{l}\text { Sin religión/ateo/ } \\
\text { agnóstico }\end{array}$ & Sí & No & Otros & Desempleado \\
\hline Daniel & 26 & Parda & CUR & $\begin{array}{l}\text { Pareja } \\
\text { casual }\end{array}$ & Católico no praticante & No & No & $\begin{array}{l}\text { Profesionales } \\
\text { de las ciencias } \\
\text { y de las artes }\end{array}$ & $\begin{array}{l}\text { Analista de } \\
\text { sistemas }\end{array}$ \\
\hline Marco & 27 & Branca & SP & $\begin{array}{l}\text { Pareja } \\
\text { casual }\end{array}$ & Católico no praticante & Sí & No & $\begin{array}{l}\text { Profesionales } \\
\text { de las ciencias } \\
\text { y de las artes }\end{array}$ & $\begin{array}{c}\text { Contabilidad en } \\
\text { una empresa de } \\
\text { publicidad }\end{array}$ \\
\hline lago & 29 & Branca & POA & $\begin{array}{l}\text { Pareja } \\
\text { casual }\end{array}$ & $\begin{array}{c}\text { Sin religión/ateo/ } \\
\text { agnóstico }\end{array}$ & No & No & Otros & Autónomo \\
\hline Michael & 29 & Branca & CUR & $\begin{array}{l}\text { Pareja } \\
\text { casual }\end{array}$ & Católico no praticante & No & No & $\begin{array}{l}\text { Profesionales } \\
\text { de las ciencias } \\
\text { y de las artes }\end{array}$ & $\begin{array}{l}\text { Desarrollador de } \\
\text { sistemas }\end{array}$ \\
\hline Damião & 31 & $S / 1$ & FOR & $\begin{array}{l}\text { Pareja } \\
\text { casual }\end{array}$ & $\begin{array}{l}\text { Sin religión/ateo/ } \\
\text { agnóstico }\end{array}$ & $S / I$ & $S / I$ & $\begin{array}{l}\text { Profesionales } \\
\text { de las ciencias } \\
\text { y de las artes }\end{array}$ & $\begin{array}{l}\text { Analista de } \\
\text { sistemas }\end{array}$ \\
\hline Cirilo & 33 & Parda & POA & $\begin{array}{l}\text { Pareja } \\
\text { estable }\end{array}$ & $\begin{array}{l}\text { Sin religión/ateo/ } \\
\text { agnóstico }\end{array}$ & Sí & No & Otros & Autónomo \\
\hline Lincon & 36 & Branca & CUR & $\begin{array}{l}\text { Pareja } \\
\text { casual }\end{array}$ & Católico no praticante & No & No & $\begin{array}{l}\text { Profesionales } \\
\text { de las ciencias } \\
\text { y de las artes }\end{array}$ & $\begin{array}{l}\text { Coordinador } \\
\text { gastronómico }\end{array}$ \\
\hline Celso & 38 & Parda & RIB & $\begin{array}{l}\text { Pareja } \\
\text { casual }\end{array}$ & Católico no praticante & Sí & Sí & Comercio & $\begin{array}{l}\text { Sector de } \\
\text { combustibles }\end{array}$ \\
\hline Evandro & 41 & Negra & POA & $\begin{array}{c}\text { Pareja } \\
\text { estable } \\
\text { seropositiva }\end{array}$ & Espiritista y otras & Sí & No & Servicios & Masoterapeuta \\
\hline Sarmento & 41 & Parda & SP & $\begin{array}{l}\text { Pareja } \\
\text { casual }\end{array}$ & $\begin{array}{c}\text { Cristiano o cree en } \\
\text { Dios }\end{array}$ & Sí & Sí & Comercio & $\begin{array}{l}\text { Importación/ } \\
\text { exportación }\end{array}$ \\
\hline Antonio & 43 & Branca & CUR & $\begin{array}{l}\text { Pareja } \\
\text { casual }\end{array}$ & Católico praticante & Sí & Sí & $\begin{array}{l}\text { Profesionales } \\
\text { de las ciencias } \\
\text { y de las artes }\end{array}$ & Abogado \\
\hline Victor & 46 & Blanca & RIB & $\begin{array}{l}\text { Pareja } \\
\text { casual }\end{array}$ & Católico no praticante & No & Sí & Comercio & Librero \\
\hline Nelson & 58 & Blanca & $\mathrm{POA}$ & $\begin{array}{l}\text { Pareja } \\
\text { casual }\end{array}$ & Católico praticante & No & Sí & Otros & $\begin{array}{l}\text { Desempleado/ } \\
\text { jubilado }\end{array}$ \\
\hline
\end{tabular}

Fuente: Información extraída de las entrevistas. S/I: Sin información; CUR: Curitiba (Paraná); POA: Porto Alegre (Rio Grande do Sul); SP: São Paulo (São Paulo); RIB: Ribeirão Preto (São Paulo); FOR: Fortaleza (Ceará).

métodos y estrategias de prevención del VIH que componen y ordenan las prácticas afectivo-sexuales de los entrevistados y, luego, se exploran sus percepciones de riesgo en el contexto de la exposición y la decisión de buscar la profilaxis posexposición sexual consentida. Denominamos "métodos de prevención" al conjunto de insumos físicos proyectados para minimizar el riesgo de las personas (condones, profilaxis preexposición y posexposición sexual, por ejemplo), mientras que las "estrategias de prevención" se refieren a la adopción de actitudes y comportamientos que también anhelan controlar los riesgos de infección, pero no necesariamente asociados al uso de insumos físicos. Los métodos y estrategias no son autoexcluyentes y las personas suelen adoptarlos de forma simultánea.

El condón masculino fue el protagonista en el repertorio de los métodos de 
prevención, no solo al VIH y a las infecciones sexualmente transmisibles, sino también para evitar embarazos indeseados. La alta protección del condón, como también la gama de patologías prevenibles con su uso fueron las principales motivaciones de su elección entre el conjunto de los insumos de prevención. Algunos relatos ilustran, de modo contundente, cómo los participantes ven el condón en el contexto de las relaciones sexuales:

Entrevistadora: ...en el último año ¿de qué manera buscaste prevenirte de la infección por VIH?

Dario (23 años, blanco): Con preservativo, básicamente.

Entrevistadora: ...quería saber ¿de qué maneras buscás prevenirte contra el VIH? Celso (38 años, pardo): Preservativo. No veo otra forma.

Diversos estudios investigaron las motivaciones y la frecuencia de uso del condón masculino por parte de los hombres en general, independientemente de la orientación sexual, o de forma comparativa entre los hombres heterosexualmente activos versus hombres que tienen sexo con hombres. Los hallazgos muestran que la utilización de este método en las prácticas sexuales es significativa, pero también que está influenciada por diversos aspectos contextuales y situacionales, como el perfil de la pareja (usuarias de drogas o trabajadoras sexuales, por ejemplo), o el tipo de la relación (estable o casual), fatores estéticos (que el pene "quede feo") y de confort (sensación de compresión o irritación). Tanto los problemas relacionados con el desempeño sexual y la calidad de la erección, como con problemas emocionales (depresión y ansiedad), ya fueron señalados como barreras para la adhesión al método. Además, desde la óptica de los hombres heterosexuales, el uso del condón con la pareja estable puede revelar infidelidad y complicar la dinámica de la relación ${ }^{(25,26,27,28,29,30,31)}$.

Otros métodos de prevención, como el gel lubricante y el condón femenino fueron mencionados solo por dos entrevistados, lo que indica poca inserción de estos insumos en el cotidiano de la prevención. Para uno de ellos (Evandro), el énfasis en proponer de forma recurrente el condón femenino a su pareja, incluso en contra de ella, se da por el hecho de que ella es seropositiva y que él posea muchas experiencias negativas con el uso del condón.

Un estudio brasileño, realizado con matrimonios heterosexuales, concluyó que, desde la óptica de los hombres, el condón femenino posee más beneficios que el masculino, aunque no sea "romántico", discreto, ni práctico ${ }^{(32)}$. Otro estudio destaca el alto costo del condón femenino, que llega a ser 21 veces más caro comparado al condón masculino, como barrera para su incorporación por parte de los matrimonios heterosexuales ${ }^{(27)}$.

La combinación de métodos (condón masculino) con estrategias de prevención surgió en varios relatos. La prueba serológica de VIH, por ejemplo, asumió tanto un carácter de prerrequisito para dejar de usar el condón masculino, de manera combinada a la consolidación del estatus de relación estable y monogámica, en cuanto a un carácter de certificado de que el individuo "no tenía nada" y que, por eso, el condón podría ser dispensado en las relaciones sexuales con parejas ocasionales. Adicionalmente, la prueba serológica de VIH también fue relatada como método preventivo a ser utilizado cuando otros métodos fallan como, por ejemplo, la rotura del condón, y fue mencionado como una buena alternativa cuando el uso de condón es indeseado.

Estos dados corroboran los hallazgos de otras investigaciones sobre el uso de la prueba serológica de VIH como prerrequisito para el abandono del condón masculino. Una investigación realizada con jóvenes y adultos de Brasil, concluyó que las motivaciones frecuentemente relatadas por hombres heterosexuales con relación a la prueba estaban frecuentemente asociadas al estatus de relacionamiento estable y a la presuposición de estabilidad y exclusividad del matrimonio, que eximía del uso del condón masculino ${ }^{(27)}$. Un estudio cualitativo realizado con 
matrimonios heterosexuales en EEUU, que realizó grupos focales, analizó la viabilidad y la aceptabilidad de un proyecto de testeo y asesoramiento de matrimonios y encontró altos niveles de disposición para la utilización de esta estrategia por parte de los participantes, así como para la discusión de acuerdos sexuales al interior del matrimonio; condiciones necesarias para el éxito de este método ${ }^{(33)}$.

En la combinación entre métodos de prevención se destacó la relación entre el no uso o la falla del condón y la búsqueda por profilaxis posexposición sexual consentida. En otras palabras, siendo el condón masculino el principal método preventivo, su falla o ausencia en las relaciones fue el principal y la más recurrente motivación de los participantes para el uso de la profilaxis, emergiendo con distinta intensidad en todas las entrevistas.

Entrevistadora: En este último año ¿cómo
te cuidaste de las enfermedades de trans-
misión sexual en general?
Marco ( 27 años, blanco): Ah, con preser-
vativo. Tanto que yo tomé [profilaxis po-
sexposición sexual consentida] porque
se rompió el preservativo.

Percepciones sobre la calidad del condón mostraron estar directamente ligadas a la eficacia del método. La (supuesta) baja calidad de los condones distribuidos gratuitamente fue descrita como la principal causa de su rotura o incluso de optar por no usarlo durante las relaciones sexuales. La resistencia o rechazo al uso del condón distribuido gratuitamente no es una exclusividad del ámbito brasileño sino que estudios internacionales investigaron las barreras relacionadas a este tipo de insumo ${ }^{(31)}$. Un participante relató que buscaría en Internet otras marcas de condón más eficaces. Algunos otros relatos señalan la baja adhesión al condón masculino debido a experiencias de rotura durante el acto sexual. En uno de los relatos, por ejemplo, el entrevistado llegó a presentar el condón roto en el momento de la selección con el equipo de salud de la unidad de atención.
Entrevistadora: Ese preservativo [que se rompió] es...

Evandro (41 años, negro): Es de acá del centro de salud. Además de la dificultad que tuvimos para desenrollarlo, y ella no me ayuda en nada. No tenés que quedarte... desenrollando, qué sé yo, parece que estás intentando colocar un cobertor de lluvia en un poste, ¿no? No da.

La literatura científica sobre percepción y gerenciamiento del riesgo a la infección por VIH es extensa y destaca la asociación de las estrategias con los métodos preventivos. Dentro de las estrategias, evitar relaciones sexuales extraconyugales, relacionamientos no monogámicos y con parejas poco conocidas, reducción del número de parejas y evitar determinados lugares en la búsqueda de relaciones sexuales casuales son estrategias recurrentes tanto en la bibliografía como en nuestros hallazgos. Veamos el siguiente relato:

Entrevistadora: En el último año ¿de qué manera buscaste prevenir la infección por VIH?

Antonio (43 años, blanco): Evitando relaciones fuera del casamiento, ¿no? Nosotros nos vamos a casar por civil, pero para evitar relaciones por fuera. Tuve una relación con una prostituta, pero usando forro [condón], ahí el domingo a la noche... ahí... tuve esa falla con una persona que conocí en la calle y... fue sin forro, entonces, ahí fue el error, ¿no?, el susto.

Estar en una relación estable y monogámica se considera una estrategia válida de prevención a la infección por VIH, sustentada en la lógica de que el riesgo sería menor porque se parte del (supuesto) presupuesto de monogamia, asentado en el ideal de fidelidad ("ninguno de los dos va a saltar la cerca"). Otros estudios señalan que las representaciones sobre fidelidad y uso de condón solo en las relaciones extraconyugales forman parte de la lógica de la prevención entre hombres heterosexuales, del mismo modo que la percepción de riesgo es baja en relaciones exclusivas, como si el peligro no pudiera venir "de adentro"(34). 
La misma representación colectiva entre los hombres heterosexuales, que pregona el uso del condón en relaciones sostenidas "fuera de casa" o "en la calle", por ser más arriesgados, también sustenta como peligrosas las parejas sexuales mantenidas ocasionalmente o con personas desconocidas. Además, seleccionar el tipo y el perfil de la pareja sexual (trabajadora sexual o usuaria de drogas, por ejemplo), así como mantener un número reducido de parejas sexuales también se destacaron en los relatos como estrategias de prevención frecuentes.

Dos participantes relataron que reducían la intensidad o evitaban determinadas prácticas sexuales como forma de disminuir el riesgo de exposición al VIH. Una mayor atención a los preliminares (evitando roces excesivos y contacto con secreciones de la pareja) durante el acto sexual también surgió en uno de los relatos como forma de manejar el riesgo de exposición.

También destacamos en varios relatos que la sensación de protección e invulnerabilidad al VIH estuvo relacionada con los ideales de masculinidad hegemónica heteronormativa. Algunos entrevistados sitúan al "otro" como vulnerable o susceptible a la infección, por no cuidarse debidamente o porque sus prácticas los colocan en esa posición de "fragilidad" y "exposición". Lincon, por ejemplo, rescató la noción de grupos de riesgo, en la figura del homosexual y del usuario de drogas, que fue predominante en la primera década de la epidemia de VIH, como los únicos o los más expuestos al virus.

Lincon (26 años, blanco): Sí, nos quedamos, quedamos... pensando ino?, en la vida, a pesar de que yo no... nunca busqué... sabemos que los mayores contagios... que los mayores contagios se dan entre los homosexuales, ¿no?, drogas y homosexuales, ¿no? Principalmente por el sexo anal, ¿no?

Además de este sentido atribuido a la invulnerabilidad masculina frente al VIH, el abandono o el rechazo del condón masculino en las relaciones estables y monogámicas, o el hecho de valerse de estrategias cuya efectividad de prevención es poco reconocida, como seleccionar parejas por sus atributos estéticos, son también ejemplos de cómo los hombres heterosexualmente activos, bajo el efecto de las normativas asociadas a la masculinidad hegemónica, expresan ideas de protección al VIH.

Las percepciones de riesgo en el contexto de la exposición y la toma de decisión pela busca de la profilaxis posexposición sexual estuvieron fuertemente relacionadas con el no uso o la falla del condón masculino, en alguna parte o en toda la práctica sexual (por rotura o irritación, por ejemplo) concomitante al tipo de pareja (relaciones casuales o con trabajadoras sexuales) y a las formas de relacionamiento (extraconyugales). Adicionalmente, la presencia del alcohol fue un factor que potenció la percepción de riesgo a la infección.

La falla del condón masculino en relaciones con trabajadoras sexuales y parejas casuales poco conocidas constituyó un notorio motivo generador de duda e inseguridad, que impulsó la búsqueda de ayuda. Uno de los entrevistados relató preocupación por las "conversaciones" sobre el supuesto alto número de parejas sexuales que su pareja teve antes dele. En sus palabras, "[...] si conmigo lo hizo sin [condón], con otros también puede haber estado".

Entrevistadora: ¿Por qué creés que estar con ella sin condón era tan riesgoso para vos al punto de que te preocupes y busques el servicio?

Iván (22 años, pardo): Porque, es así, yo no conozco a la persona. Primero, yo no la conozco, ¿entendés? Ahí, después, escuché ciertas cosas ¿entendés? Vino a hablarme. Ahí, ella ya había estado con otros ¿entendés? Entonces, ahí me quedé medio preocupado, ¿no? Porque si conmigo estuvo sin, con otro también puede haber estado.

En otro relato quedó en evidencia el papel de los factores contextuales (lugar donde sucedió la exposición, por ejemplo) cuando se 
asocian a la falla del condón, para potencializar la percepción de riesgo de hombres heterosexualmente activos analizados.

Lincon (26 años, blanco): nos encontramos en un hotelito así, hotelito.

Entrevistadora: ¿Y no la conocías a ella? Lincon (26 años, blanco): Ah, más o menos, así, medio de vista, arreglamos por teléfono y tal, solo que ahí, conversando con un amigo mío, ya sabíamos que esa chica podía tener alguna cuestión de trabajo sexual, esas cosas, entonces por eso que terminé buscando.

En cuanto al incremento de la percepción de riesgo vinculado al uso de alcohol, seis entrevistados mencionaron que ingirieron alguna sustancia antes o durante el sexo, pero solo la mitad de estos destacó que el uso influyó en cómo ellos se previnieron durante el contexto de exposición sexual que los hizo buscar la profilaxis posexposición sexual. En palabras de Antonio: "El alcohol realmente complicó las cosas [...] fue una... estupidez, una tontería, un minuto de estupidez, de hecho".

Una revisión bibliográfica, realizada entre 2000 y 2007, concluyó que el uso de alcohol, cuando se combina con prácticas sexuales, se mostró como factor de riesgo para infecciones de transmisión sexual/sida, aumento del número de parejas concomitantes y no uso de condón. Sin embargo, esa misma investigación concluyó que, en general, los hombres que tienen sexo con hombres, adolescentes y profesionales del sexo poseen mayor prevalencia de sexo bajo el efecto del alcohol que las personas en general, y que los hombres poseen mayores prevalencias que las mujeres ${ }^{(35)}$.

Por último, vale destacar que, en el momento de las entrevistas, todos los participantes ya conocían la existencia de la profilaxis y muchos ya tenían información sobre cómo actúan los antirretrovirales y cuáles son los mecanismos de protección que ofrece la profilaxis posexposición sexual. Sin embargo, la mitad de los participantes no tenía conocimientos sobre la profilaxis posexposición sexual previamente a la exposición sexual de riesgo, lo que dificultó el acceso y aumentó el itinerario recorrido hasta el servicio de salud en el cual hizo uso de la profilaxis.

Buscar ayuda e información especializada, sea en unidades de salud o en websites, después de situaciones de exposición sexual de riesgo de infección al VIH puede ser considerado una estrategia preventiva en sí. Nuestros hallazgos indican que, tanto los participantes que tengan algún conocimiento, aunque limitado, como para aqueIlos que no conocían la profilaxis, buscaron información sobre cómo actuar luego de la exposición, sobre todo en Internet.

\begin{abstract}
Michael (29 años, blanco): En realidad, yo quedé... yo busqué información ¿no?, el $90 \%$ de las personas que pasan por una situación así buscan información en Internet, tal vez no sea lo más confiable, pero fue a través de Internet que descubrí este lugar, ¿no? Y en cuanto lo descubrí, ya decidí en el momento... Ahí en la propia página ya vi que tenía esta posibilidad de tratamiento, entonces, pensé: "bueno, voy a ver primero, para evaluar la situación y si la médica me manda a tomar algún remedio, voy a tomar el remedio".
\end{abstract}

De hecho, la utilización de Internet y de medios de comunicación masiva para tener acceso a conocimientos sobre métodos y estrategias de prevención y tratamiento del $\mathrm{VIH}$ o infecciones de transmisión sexual es una práctica común y ya fue mostrada por una investigación cualitativa brasileña en la cual los hombres más viejos y menos escolarizados eran también los menos informados sobre infecciones de transmisión sexual o sida al ser comparados con los más jóvenes ${ }^{(27)}$.

Los entrevistados que no conocían la profilaxis posexposición sexual anteriormente al episodio de la exposición (ocho) reflejan el bajo acceso a la información sobre prevención a la infección por VIH o enfermedades de transmisión sexual en los hombres heterosexualmente activos. En diferentes estudios también se constató que este segmento no solo posee menos información sobre los métodos y estrategias de prevención, 
sino también acceden menos a determinados insumos, como la prueba serológica de $\mathrm{VIH}^{(36,37,38)}$. Esto se debe, en parte, a la influencia de los patrones de masculinidad en el bajo reconocimiento del riesgo y en la baja movilización de los heterosexuales frente a la epidemia del VIH desde su surgimiento, lo que hace que, hasta hoy, los hombres heterosexuales no posean redes de apoyo y socialización en las que circule información sobre nuevas tecnologías preventivas ${ }^{(39)}$.

\section{CONSIDERACIONES FINALES}

En este estudio, que exploró las percepciones de riesgo y prevención al VIH entre hombres heterosexualmente activos en un contexto de prevención combinada, con énfasis en la utilización de profilaxis posexposición sexual, sobresalieron aspectos subjetivos y socioculturalmente marcados por género y orientación sexual que proveen elementos importantes para pensar cómo los ideales relacionados con la masculinidad hegemónica influyen en cómo los hombres heterosexuales piensan y se previenen de la infección por $\mathrm{VIH}$ en el contexto actual de la epidemia.

Para Connell y Pearse ${ }^{(40)}$, las relaciones de género se producen en las interacciones y por medio de las prácticas ejecutadas en el cotidiano de las personas. Esas prácticas, a pesar de suceder y ser producidas a nivel de las microrelaciones, reproducen ideales colectivos de determinada sociedad y son históricamente situadas; luego, son modificadas y ajustadas en diferentes contextos y para determinados segmentos de hombres $y$ mujeres. El entendimiento entre los hombres heterosexualmente activos participantes de la investigación de que el sida consiste en una "enfermedad de los otros" repercute en cómo perciben su lugar en el contexto actual de la epidemia, tornándolos también vulnerables a la infección por la noción de "no pertenencia" a un "grupo de riesgo"(4,5,28).

Los marcos referenciales de masculinidades y feminidades apuntan a que la prevención no solo del VIH, sino también de las otras infecciones de transmisión sexual, sea difícil para los hombres y mujeres. En lo referente a los hombres heterosexualmente activos, por ejemplo, vimos que la percepción y el gerenciamiento de riesgo están arraigados en bases contextuales que envuelven aspectos como estética y comportamiento sexual previo de la pareja, tipo de pareja (conocida o desconocida) y tipo de vínculo (exclusivo o paralelo), además de prerrogativas sobre la ocupación (profesional del sexo) de las parejas.

El conjunto de los aspectos considerados por los hombres heterosexualmente activos en la percepción y el gerenciamiento del riesgo de la infección por VIH también dialoga directamente con la jerarquización del riesgo, o sea, con los modos cómo las personas manejan los elementos negativos asociados a los riesgos, como también los beneficios que pueden surgir cuando asumen vivenciarlos. Todas esas cuestiones son fruto del proceso reflexivo de nuestro tiempo ${ }^{(41)} \mathrm{y}$ están vinculados a las posiciones de los sujetos en los sistemas de orden de género y sexualidad, pero también de clase, raza, etnia, generación, entre otros.

El protagonismo del condón masculino en las narrativas de prevención del VIH y, al mismo tiempo, su falla, señalada como la principal motivación para decidir buscar la profilaxis posexposición sexual, demuestran la complejidad de la relación entre riesgo y placer, lo que ha sido destacado como un tema a ser explorado en las investigaciones de género y sexualidad en el campo del $\mathrm{VIH}$ sida $^{(2,4,5)}$. Además, ciertos estudios cualitativos sobre las representaciones del uso del condón para hombres sugieren que los ideales hegemónicos de masculinidad, creencia en la fidelidad femenina, percepciones de que el condón influye en la potencia o en la performance sexual son argumentos frecuentemente relatados ${ }^{(25,28,30,31)}$, que corroboran los hallazgos de la presente investigación.

Por último, ante el actual escenario de la epidemia de $\mathrm{VIH}$, se torna urgente el abandono de la noción, aún muy presente en el sentido común y en buena parte de la bibliografía científica, de que el segmento de los hombres heterosexuales consiste en un 
grupo homogéneo, que comparte características idénticas entre sí, lo que llevaría, por lo tanto, a la invisibilidad de estos sujetos en las políticas y programas de prevención de la infección por $\mathrm{VIH}^{(3,5)}$. Además, las características relacionadas con las masculinidades están condicionadas a los diferentes contextos y de maneras diversas cuando se consideran marcadores sociales de la diferencia ${ }^{(40,42)}$. Evidenciar, por lo tanto, el seguimiento de los hombres heterosexualmente activos, en especial en el contexto del escenario actual de prevención al VIH que incluye los métodos biomédicos basados en terapia antirretroviral, es también visibilizarlos en futuras políticas y programas del área, como también (re)conocer las vulnerabilidades que los atraviesan en el contexto personal-colectivo de la prevención, marcados por el género y la orientación sexual| ${ }^{(3,5,43)}$.

\section{AGRADECIMENTOS}

La investigación fue financiada por el Ministério da Saúde de Brasil, código 027941/2012, y por el Conselho Nacional de Desenvolvimento Científico y Tecnológico (CNPq), Edital Universal, código $\mathrm{N}^{\circ}$ 456551/2014-0.

\section{REFERENCIAS BIBLIOGRÁFICAS}

1. Brasil, Ministério da Saúde. Boletim Epidemiologico de Hepatites Virais [Internet]. 2018 [citado 18 dic 2018]. Disponible en: https://tinyurl.com/ t7zfbu8.

2. Dworkin SL. Men at risk: Masculinity, heterosexuality, and HIV prevention. New York: NYU Press; 2015.

3. Couto MT, Grangeiro A, Venturi G, Levy RB. Rendering visible heterosexually active men in Brazil: A national study on sexual behaviour, masculinities and HIV risk. Current Sociology. 2018;66(5):704723.

4. Higgins JA, Hoffman S, Dworkin SL. Rethinking gender, heterosexual men, and women's vulnerability to HIV/AIDS. American Journal of Public Health. 2010;100(3):435-445.

5. Leal AF, Knauth DR, Couto MT. The invisibility of heterosexuality in HIV/AIDS prevention for men. Revista Brasileira de Epidemiologia. 2015;18(Suppl 1):S143-S155.

6. Connell R. Gender, health and theory: Conceptualizing the issue, in local and world perspective. Social Science \& Medicine. 2012;74(11):16751683.

7. Fleming PJ, DiClemente RJ, Barrington C. Masculinity and HIV: Dimensions of masculine norms that contribute to men's HIV-related sexual behaviors. AIDS and Behavior. 2016;20(4):788-798.
8. Connell R. Masculinities. Cambridge: Polity; 2005.

9. Gomes R. Sexualidade masculina, gênero e saúde. Rio de Janeiro: Editora Fiocruz; 2008.

10. Grangeiro A, Kuchenbecker R, Veras MA. New HIV prevention methods: recognizing boundaries between individual autonomy and public policies. Revista Brasileira de Epidemiologia. 2015;18(Suppl 1):S1-S4.

11. Rotheram-Borus MJ, Swendeman D, Chovnick G. The past, present, and future of HIV prevention: integrating behavioral, biomedical, and structural intervention strategies for the next generation of HIV prevention. Annual Review of Clinical Psychology. 2009;5:143-167.

12. Brasil, Ministério da Saúde. Prevenção combinada do HIV: Bases conceituais para profissionais, trabalhadores(as) e gestores(as) de saúde. Brasília: Ministério da Saúde; 2017.

13. UNAIDS. Combination HIV prevention: tailoring and coordinating biomedical, behavioural and structural strategies to reduce new HIV infections. Geneva: UNAIDS; 2010.

14. UNAIDS. Guia de terminologia do UNAIDS. Brasília: UNAIDS; 2017.

15. Brasil, Ministério da Saúde. Protocolo clínico e diretrizes terapêuticas para hepatite C e coinfecções. Brasília: Ministério da Saúde; 2017.

16. Maksud I, Fernandes NM, Filgueiras SL, Maksud I, Fernandes NM, Filgueiras SL. Technologies for HIV prevention and care: challenges for health services. Revista Brasileira de Epidemiologia. 2015;18(Suppl 1):S104-S119.

17. Otten RA, Smith DK, Adams DR, Pullium JK, Jackson E, Kim CN, et al. Efficacy of postexposure prophylaxis after intravaginal exposure of pig-tailed macaques to a human-derived retrovirus (Human 
Immunodeficiency Virus Type 2). Journal of Virology. 2000;74(20):9771-9775.

18. Tsai CC, Follis KE, Sabo A, Beck TW, Grant RF, Bischofberger N, et al. Prevention of SIV infection in macaques by (R)-9-(2-phosphonylmethoxypropyl) adenine. Science. 1995;270(5239):1197-1199.

19. Fernández-Balbuena S, Belza MJ, Castilla J, Hoyos J, Rosales-Statkus ME, Sánchez R, et al. Awareness and use of nonoccupational HIV post-exposure prophylaxis among people receiving rapid HIV testing in Spain. HIV Medicine. 2013;14(4):252-257.

20. Mehta SA, Silvera R, Bernstein K, Holzman RS, Aberg JA, Daskalakis DC. Awareness of post-exposure HIV prophylaxis in high-risk men who have sex with men in New York City. Sexually Transmitted Infections. 2011;87(4):344-348.

21. Grangeiro A, Couto MT, Peres MF, Luiz O, Zucchi EM, De Castilho EA, et al. Pre-exposure and postexposure prophylaxes and the combination HIV prevention methods (The Combine! Study): Protocol for a pragmatic clinical trial at public healthcare clinics in Brazil. BMJ Open. 2015;5(8):1-11.

22. Minayo MCDS, Deslandes SF, Neto OC, Gomes R. Pesquisa social: Teoria, método e criatividade. Rio de Janeiro: Editora Vozes; 2011.

23. Ricouer P. Tempo e narrativa (Tomo III). Campinas: Papirus; 2012.

24. Gomes R, Souza ER, Minayo MCS, Malaquias JV, Silva CFR, et al. Organização, processamento, análise e interpretação de dados: o desafio da triangulação. En: Avaliação por triangulação de métodos: abordagem de programas sociais. Rio de Janeiro: Fiocruz; 2005.

25. Dourado I, MacCarthy S, Reddy M, Calazans G, Gruskin S. Revisiting the use of condoms in Brazil. Revista Brasileira de Epidemiologia. 2015;18(Suppl 1):S63-S88.

26. Costa EC V, Ribeiro D, Correia P. Associação entre estados afetivos negativos e comportamentos sexuais de risco. Revista de Estudios e Investigación en Psicología y Educación. 2017;(14):206-209.

27. Garcia S, Souza FM. Vulnerabilidades ao HIV/ aids no contexto brasileiro: Iniquidades de gênero, raça e geração. Saúde e Sociedade. 2010;19(Suppl. 2):9-20.

28. Gomes R, Souza ACB, Rebello LEFS. Homens e a prevenção da aids: análise da produção do conhecimento da área da saúde. Interface - Comunicação, Saúde, Educação. 2011;15(36):67-78.
29. Guerriero I, Ayres JRC, Hearst N. Masculinidade e vulnerabilidade ao HIV de homens heterossexuais, São Paulo, SP. Revista de Saúde Pública. 2002;36(4 suppl):50-60.

30. Hill BJ, Sanders SA, Crosby RA, Ingelhart KN, Janssen E. Condom-associated erection problems: Behavioural responses and attributions in young, heterosexual men. Sexual Health. 2015;12(5):397404.

31. Kanda L, Mash R. Reasons for inconsistent condom use by young adults in Mahalapye, Botswana. African Journal of Primary Health Care \& Family Medicine. 2018;10(1):e1-7.

32. Gomes VL de O, Fonseca AD da, Jundi $M$ da $G$, Severo TP, de Oliveira Gomes VL, da Fonseca AD, et al. Percepçõeses de casais heterossexuais acerca do uso da camisinha feminina. Escola Anna Nery. 2011;15(1):22-30.

33. Stephenson R, Finneran C, Goldenberg $T$, Coury-Doniger $P$, Senn TE, Urban $M$, et al. WiIlingness to use couples HIV testing and discussion of sexual agreements among heterosexuals. Springerplus. 2015;4(1):169.

34. Silva CGM. O significado de fidelidade e as estratégias para prevenção da Aids entre homens casados. Revista de Saúde Pública. 2002;36(Suppl. 4):S40-S49.

35. Cardoso LRD, Malbergier A, Figueiredo TFB. O consumo de álcool como fator de risco para a transmissão das DSTs/HIV/Aids. Archives of Clinical Psychiatry (São Paulo). 2008;35(Suppl. 1):S70-S75.

36. Dennison O, Wu Q, Ickes M. Prevalence of human immunodeficiency virus testing and associated risk factors in college students. Journal of American of College Health. 2014;62(5):309-318.

37. McElrath K, Stana A, Taylor A, Johnson-Arnold $\mathrm{L}$. Race/sex interactions and HIV testing among college students. Journal of Racial and Ethnic Health Disparities. 2016;4(1):112-121.

38. Thomas J, Harden A. Methods for the thematic synthesis of qualitative research in systematic reviews. BMC Medical Research Methodology. 2008;8:45.

39. Walters SM, Rivera AV, Starbuck L, Reilly $\mathrm{KH}$, Boldon N, Anderson BJ, et al. Differences in awareness of pre-exposure prophylaxis and post-exposure prophylaxis among groups at-risk for HIV in New York State: New York City and Long Island, NY, 2011-2013. Journal of Acquired Immune Deficiency Syndromes. 2017;75(Suppl 3):S383-S391. 
40. Connell R, Pearse R. Gênero: uma perspectiva global, compreendendo o gênero da esfera pessoal à política no mundo contemporâneo. São Paulo: nVersos; 2015.

41. Perrusi A, Franch M. Carne com carne: gestão do risco e HIV/Aids em casais sorodiscordantes no Estado da Paraíba. Revista de Ciências Sociais Política \& Trabalho. 2012;2(37):179-200.
42. Courtenay WH. Constructions of masculinity and their influence on men's well-being: A theory of gender and health. Social Science \& Medicine. 2000;50(10):1385-1401.

43. Gomes R, Schraiber LB, Couto MT, Valença OAA, da Silva GSN, Figueiredo WS, et al. O atendimento à saúde de homens: Estudo qualitativo em quatro estados brasileiros. Physis. 2011;21(1):113128.

\section{FORMA DE CITAR}

Santos LA, Couto MT, Mathias A, Grangeiro A. Hombres heterosexualmente activos, masculinidades, prevención de infección por VIH y búsqueda de profilaxis posexposición sexual consentida. Salud Colectiva. 2019;15:e2144. doi: 10.18294/sc.2019.2144.

Recibido: 22-01-2019 | Versión final: 21-11-2019 | Aprobado: 22-11-2019

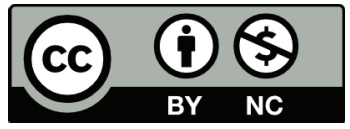

Esta obra está bajo una licencia de Creative Commons Reconocimiento-NoComercial 4.0 Internacional. Reconocimiento - Permite copiar, distribuir y comunicar públicamente la obra. A cambio, se debe reconocer y citar al autor original. No Comercial - Esta obra no puede ser utilizada con finalidades comerciales, a menos que se obtenga el permiso. 